BMJ Paediatrics Open

\title{
A Prospective, observational cohort study to identify neonates and children at risk of postdischarge mortality in Dar es Salaam, Tanzania and Monrovia, Liberia: the PPDM study protocol
}

Chris A Rees (D) , ${ }^{1,2}$ Rodrick Kisenge, ${ }^{3}$ Readon C Ideh, ${ }^{4}$ Julia Kamara, ${ }^{4}$ Abraham Samma, ${ }^{3}$ Evance Godfrey, ${ }^{3}$ Hussein K Manji, ${ }^{5}$ Christopher R Sudfeld, ${ }^{6,7}$ Adrianna Westbrook, ${ }^{8}$ Michelle Niescierenko, ${ }^{9,10}$ Karim P Manji, ${ }^{3}$ Christopher P Duggan ${ }^{6,7,11,12}$

To cite: Rees $\mathrm{CA}$, Kisenge $\mathrm{R}$, Ideh RC, et al. A Prospective, observational cohort study to identify neonates and children at risk of postdischarge mortality in Dar es Salaam, Tanzania and Monrovia, Liberia: the PPDM study protocol. BMJ Paediatrics Open 2022;6:e001379. doi:10.1136/ bmjpo-2021-001379

- Additional supplemental material is published online only. To view, please visit the journal online (http://dx.doi.org/ 10.1136/bmjpo-2021-001379).

KPM and CPD contributed equally.

KPM and CPD are joint senior authors.

Received 15 December 2021 Accepted 29 December 2021

Check for updates

C Author(s) (or their employer(s)) 2022. Re-use permitted under CC BY-NC. No commercial re-use. See rights and permissions. Published by BMJ

For numbered affiliations see end of article.

Correspondence to Dr Chris A Rees; chris.rees@ emory.edu

\section{ABSTRACT}

Introduction Over half of the 5 million annual deaths among children aged 0-59 months occur in sub-Saharan Africa. The period immediately after hospitalisation is a vulnerable time in the life of a child in sub-Saharan Africa as postdischarge mortality rates are as high as $1 \%-18 \%$. Identification of neonates and children who are at highest risk for postdischarge mortality may allow for the direction of interventions to target patients at highest risk.

Methods and analysis The Predicting Post-Discharge Mortality study is a prospective, observational study being conducted at Muhimbili National Hospital (Dar es Salaam, Tanzania) and John F. Kennedy Medical Center (Monrovia, Liberia). The aim is to derive and validate two, age population specific, clinical prediction rules for the identification of neonates $(n=2000)$ and children aged 1-59 months $(n=2000)$ at risk for all-cause mortality within 60 days of discharge from the neonatal intensive care unit or paediatric ward. Caregivers of participants will receive phone calls $7,14,30,45$ and 60 days after discharge to assess vital status. Candidate predictor variables will include demographic, anthropometric and clinical factors. Elastic net regression will be used to derive the clinical prediction rules. Bootstrapped selection with repetitions will be used for internal validation. Planned secondary analyses include the external validation of existing clinical prediction models, determination of clinicians' ability to identify neonates and children at risk of postdischarge mortality at discharge, analysis of factors associated with hospital readmission and unplanned clinic visits and description of health-seeking behaviours in the postdischarge period.

Ethics and dissemination This study received ethical clearance from the Tanzania National Institute of Medical Research, Muhimbili University of Health and Allied Sciences, the John F. Kennedy Medical Center Institutional Review Board, and the Boston Children's Hospital Institutional Review Board. Findings will be disseminated at scientific conferences and as peerreviewed publications.

\section{What is already known on this topic?}

Postdischarge mortality rates among children in sub-Saharan Africa are estimated to be as high as $1 \%-18 \%$.

- Two clinical prediction rules have been derived from studies conducted in Uganda and Mozambique to identify children at risk of postdischarge mortality in sub-Saharan Africa.

Neither of the existing clinical prediction rules have been externally validated and have not specifically targeted neonates

\section{What this study hopes to add?}

This prospective, observational cohort study in Liberia and Tanzania will derive and validate two, novel, population-specific, clinical prediction rules to identify neonates and children at risk of postdischarge mortality

- This study will elucidate clinician gestalt in the identification of neonates and children at risk of postdischarge mortality

- This study will add further understanding of postdischarge health-seeking behaviours for children in sub-Saharan Africa.

\section{INTRODUCTION}

Since the 1990s, mortality rates among children under 5 years of age decreased by $>50 \%$ globally. ${ }^{1}$ However, progress in reducing childhood mortality has been geographically uneven, with more than half of the 5 million annual deaths among children occurring in sub-Saharan Africa. ${ }^{2}$ Limited access to 
healthcare providers, long distances to healthcare facilities and delayed care seeking contribute to high rates of childhood mortality in sub-Saharan Africa. ${ }^{3-5}$

Recently, emphasis has been placed on increasing access to high-quality healthcare for children in subSaharan Africa. ${ }^{6}$ However, the period immediately after hospitalisation is a vulnerable time in the life of a child in resource-limited settings. Postdischarge mortality rates among children in parts of sub-Saharan Africa are estimated to be as high as $1 \%-18 \%$, even outpacing inpatient mortality rates in some studies. ${ }^{7}$ Despite this documented vulnerability, postdischarge mortality has largely been neglected in policy and practice. ${ }^{8}$

Two clinical prediction rules have been derived to identify children at risk of postdischarge mortality in single countries in sub-Saharan Africa. Wiens et al derived a model to identify infants and children aged 6-59 months at risk of postdischarge mortality up to 6 months after discharge from two Ugandan hospitals with suspected or proven infections. ${ }^{9}$ In addition, Madrid et al derived a model to identify children aged $<15$ years admitted with all diagnoses at risk of 90-day postdischarge mortality in Mozambique. ${ }^{10}$ Only malnutrition, HIV-positive status and altered mental status were associated with postdischarge mortality in both studies. All other clinical parameters associated with postdischarge mortality differed between these two studies.

Neither of these clinical prediction rules have been externally validated, making their impact on reducing postdischarge mortality unclear. Moreover, neonates have not been the focus of either clinical prediction rule despite contributing to nearly half of all deaths among children aged $0-59$ months. ${ }^{11}$ Factors that predict postdischarge mortality for neonates may differ from those for children 1-59 months due to differences in leading causes of death by age. Identification of neonates and children who are at highest risk for postdischarge mortality may allow for the direction of interventions to target patients at highest risk. ${ }^{8}$

To this end, the Predicting Post-Discharge Mortality (PPDM) study will derive and validate two, age populationspecific, clinical prediction rules to identify neonates and children aged 1-59 months at risk of all-cause mortality within 60 days of discharge from the neonatal intensive care unit (NICU) or paediatric ward at two sites in subSaharan Africa.

\section{METHODS}

\section{Study design}

PPDM is a prospective, observational study being conducted at Muhimbili National Hospital in Dar es Salaam, Tanzania and John F. Kennedy Medical Center in Monrovia, Liberia. The study protocol was developed by collaborators in Tanzania (Muhimbili University of Health and Allied Sciences and Muhimbili National Hospital), Liberia (John F. Kennedy Medical Center) and the USA (Boston Children's Hospital, Harvard T.H. Chan
School of Public Health and Emory University School of Medicine). The study diagram is included in figure 1 . Enrolment began on 1 October 2019. Patient follow-up data collection will conclude on 31 January 2022. This protocol follows the Strengthening the Reporting of Observational Studies in Epidemiology guidelines for cohort studies (online supplemental file 1).

The combined study population between Liberia and Tanzania will be approximately 4000 participants (2000 neonates, 2000 children aged 1-59 months) who are discharged from the two sites. Each site will enrol 1000 neonates and 1000 children aged 1-59 months. Enrolled neonates and children will be followed through phone calls after discharge at 7,14, 30, 45 and 60 days to assess postdischarge outcomes and health-seeking behaviours.

\section{Patient and public involvement}

Caregivers, patients and the public were not involved in the development of the study protocol.

\section{Primary and secondary objectives}

The primary objective of the study is to derive and internally validate two, age population-specific, clinical prediction rules to identify neonates and children aged 1-59 months at risk of postdischarge mortality within 60 days of discharge from the NICU or paediatric ward at two sites in sub-Saharan Africa. Our secondary objectives are to (1) externally validate existing clinical prediction rules for postdischarge mortality developed from studies in Uganda and Mozambique, ${ }^{9} 10$ (2) determine clinicians' ability to accurately identify neonates and children at risk of postdischarge mortality and hospital readmission at the time of hospital discharge, (3) describe factors associated with hospital readmission and unplanned clinic visits after discharge and (4) describe health-seeking behaviours in the postdischarge period among children who die as well as those who survive beyond 60 days.

\section{Study setting}

Muhimbili National Hospital and John F. Kennedy Medical Center are large, referral, training hospitals for Tanzania and Liberia, respectively. Both hospitals are government hospitals supported by each country's Ministry of Health. Both hospitals are located in urban areas with surrounding populations of 4 million for Muhimbili National Hospital and 1.5 million for John F. Kennedy Medical Center. Both hospitals have approximately 140-150 paediatric discharges and approximately 170 NICU discharges per month.

\section{Eligibility}

We will include participants aged 0-59 months with all diagnoses who are discharged from the NICU or the paediatric ward. Participants will be included if their caregivers consent to have their hospital admission data gathered and to receive follow-up phone calls. We will exclude children who (1) die during their initial hospitalisation, (2) are older than 59 months of age or (3) have non-consenting caregivers. We will not enrol participants 


\section{Inpatient Pediatric Ward}

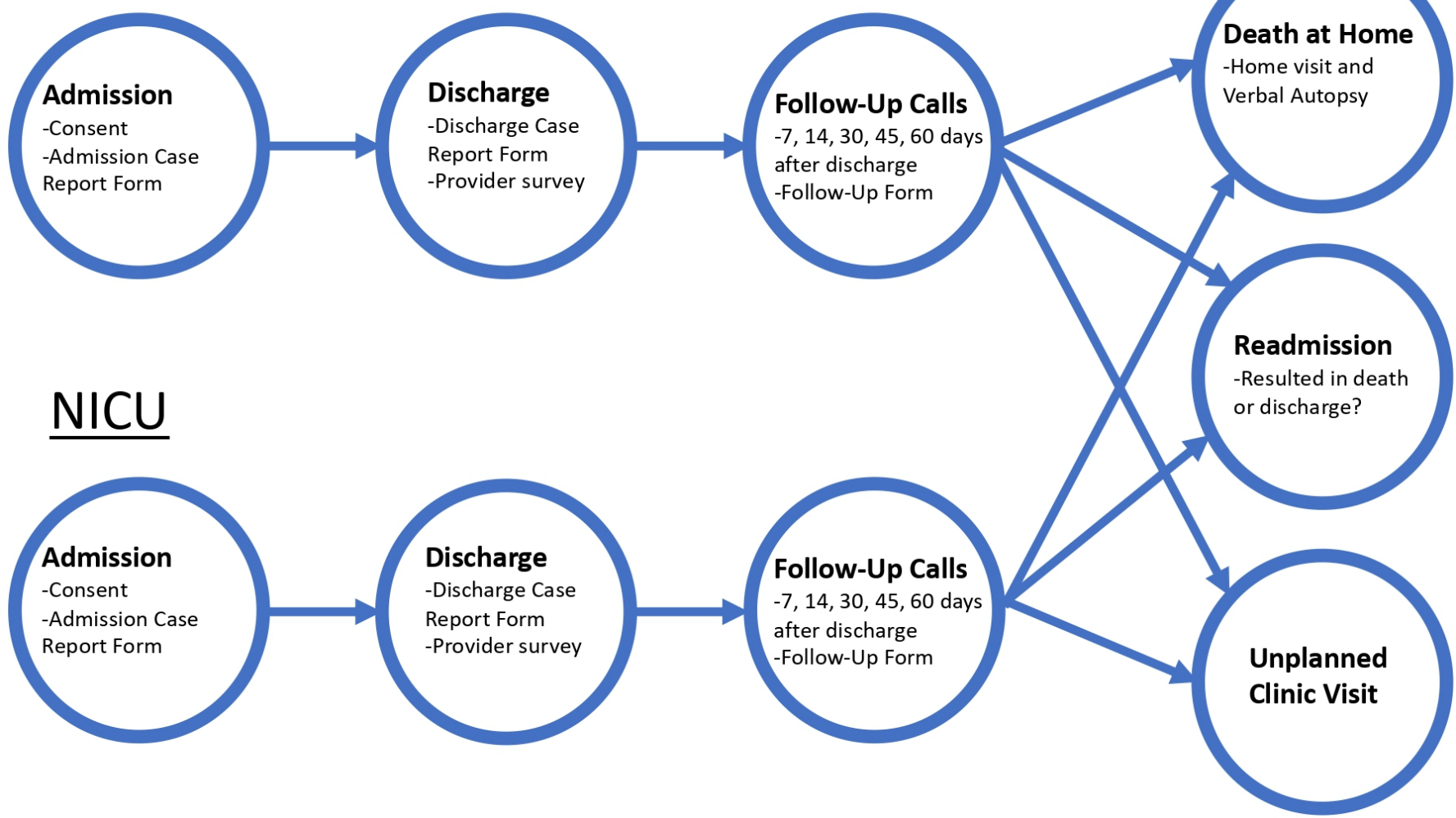

Figure 1 The Predicting Post-Discharge Mortality study design. (NICU: neonatal intensive care unit).

who are transferred to other hospitals; however, both hospitals are large referral hospitals and, therefore, transfer out is rare.

\section{Sample size}

Previous studies at Muhimbili National Hospital evaluating patients with malnutrition and abdominal pain suggest postdischarge mortality rates as high as $13 \%-15 \% .^{12} 13$ Since there are no previous studies describing postdischarge mortality at John F. Kennedy Medical Center and postdischarge mortality rates among all discharged patients at Muhimbili National Hospital may be lower than among specific populations, we conservatively estimate a $5 \%$ postdischarge mortality rate. We also anticipate a lost-to-follow-up rate of $3 \%$ in each cohort. Therefore, we will enrol 1000 patients in each cohort (NICU and paediatric wards) at each site $(n=4000$ total $)$ to derive and validate two, age population-specific, clinical prediction rules to predict postdischarge mortality (2000 in each population). We expect at least 100 deaths and 150 hospital readmissions in each cohort. Using the rule of 10 events per item in a clinical prediction rule and assuming a $5 \%$ mortality rate, we will be able to include at least five variables in each clinical prediction rule for postdischarge mortality. ${ }^{14}$

\section{Standard of care}

All enrolled children will receive standards of care per Tanzanian Ministry of Health guidelines at Muhimbili National Hospital and standards of care set forth by the John F. Kennedy Medical Center and the Liberian Ministry of Health. This care will be provided by the clinical care teams and not by this study's staff.

\section{Recruitment}

Caregivers of participants will be approached by research assistants at the time of hospital discharge and asked to consent to the study. Consenting providers will provide informed consent in their own language (written in Kiswahili or witnessed thumbprint if not literate in Tanzania and verbal consent in English in Liberia). We will collect data at the time of discharge using a secure case report form on password-protected tablets or computers. Research assistants at each site will undergo rigorous training regarding study enrolment, data collection, retention and follow-up.

\section{Screening and enrollment procedures}

Research assistants will identify consecutive patients eligible for study enrolment in the days preceding discharge. During that time, research assistants will confirm inclusion and exclusion criteria for each potential participant who is admitted to the NICU or the paediatric ward. For consenting participants, key clinical findings and laboratory data will be documented in case report forms for factors present at admission and discharge (online supplemental files 2, 3). If no contraindication to study participation is found, a research assistant will approach the primary caregiver of the child and describe the study procedures. If the child is eligible and 
the caregiver consents to participation, informed consent will be obtained. The parent or guardian must provide informed consent prior to enrolment.

Research assistants will fill out case report forms on password-protected, encrypted software (Microsoft SQL in Tanzania and Kobo Toolbox in Liberia) with variables collected as part of the study. We will collect patient anthropometrics as measured during hospitalisation using standardised scales for weight, height/length as measured using available measuring boards and middleupper arm circumference if documented by clinicians caring for children during their hospitalisation. We will calculate weight for height z-scores for age. We will collect data on documented nutritional status for the child during the hospitalisation (eg, severe acute malnutrition, kwashiorkor, etc).

Primary and secondary diagnoses at the time of admission and discharge will be collected by research assistants as documented. We will also collect information on any reported comorbidities (eg, HIV, tuberculosis, malignancies, etc) documented by the clinical care team. We will document the duration of hospital admission as documented in patients' charts. Research assistants will ask caregivers about recent hospitalisations and document responses. Research assistants will record results from labs and imaging done during the hospitalisation, receipt of therapies including oxygen (ie, nasal cannula, face mask, etc), intravenous fluids and mode of receipt (ie, bolus, maintenance fluids, etc), medications (both type and duration) received during hospitalisation and procedures or surgeries performed.

As this is an observational study, we anticipate there will be some missing variables in clinical documentation. Research assistants will attempt to fill in any missing variables prior to discharge. Variables for individual participants still missing after the research assistants' attempt to complete data will be accounted for with multiple imputations or excluded from analyses.

Research assistants will collect detailed contact information from the caregivers using a standardised patient locator form, including at least two working cell phone numbers for the caregiver, and a physical description of the whereabouts of the child's home. If a caregiver does not have a mobile phone, we will obtain the mobile phone information of a household member or friend. We expect fewer than $5 \%$ of participants to be without cell phone access.

\section{Follow-up visits}

Research assistants will make phone calls to each caregiver of children at $7,14,30,45$ and 60 days after hospital discharge to ascertain the child's vital status and to ask about health-seeking behaviours (online supplemental file 4). The equivalent of US\$1 will be transferred via local phone companies to each participant for each follow-up phone call. Text messages will be sent to caregivers who do not answer the phone. ${ }^{15}$ If caregivers are still unreachable, research assistants will conduct home visits. Participants whose caregivers are unreachable despite three phone calls, two text messages and a home visit will be classified as lost-to-follow-up.

At each telephone or in-person contact, a research assistant will ask the caregiver about the following and record the responses:

1. The child's status (ie, well, ill, hospitalised, dead).

2. Any intercurrent illnesses.

3. Any clinic or emergency department visits since hospitalisation.

4. The caregivers' seeking of other forms of treatment (ie, herbs, pharmacy, etc).

5. Reason for returning to clinic (eg, fever, vomiting, etc).

Caregivers will be asked by study staff to take their child to a clinic or hospital if they feel their children are severely ill between phone calls. For patients who are found to have died at home or in hospitals other than Muhimbili National Hospital and John F. Kennedy Medical Center prior to follow-up phone calls, research assistants will conduct home visits to use the WHO's 2016 Verbal Autopsy Tool ${ }^{16}$ (online supplemental file 5) used to describe the death of a child outside of the formal healthcare system. ${ }^{1718}$ Home visits will be arranged at a time convenient for caregivers and with adequate time after the child's death to allow the family to mourn as is culturally appropriate. Research assistants will bring a contextually appropriate condolence gift (eg, a bag of rice) to caregivers of the deceased child. All research staff who will conduct home visits will undergo rigorous training in use of the WHO's 2016 Verbal Autopsy Tool.

\section{Quality assurance}

A study coordinator will be employed to assure that training of the field staff is high quality and rigorous. A data manager will generate weekly data summaries showing recruitment progress, follow-up and event rates to ensure high-quality and complete data reporting. These quality assurance approaches will be reinforced by each site's principal investigator and by collaborators from the USA. Data cleaning will be performed using consistency and range checks. Data quality checks will also be applied weekly, and feedback will be provided to the study coordinators at both sites.

\section{Outcome measures}

Our primary outcome of postdischarge mortality and secondary outcomes including hospital readmissions, unplanned clinic visits and other care-seeking behaviours will be measured by caregiver report during follow-up calls and in-person home visits.

\section{Statistical analysis}

All candidate variables will be selected $a$ priori based on prior studies, ${ }^{7}{ }^{19-22}$ modified Delphi studies of factors thought to be associated with postdischarge mortality in sub-Saharan Africa, ${ }^{23} 24$ and experience among the research team. For the derivation of the clinical 
prediction rules, we will use elastic net regression, ${ }^{25}$ including all candidate variables to assess the strength of the association of each candidate variable on postdischarge mortality. Associations with 95\% CIs for adjusted ORs that do not cross one will be considered significant. To determine the weighted points assigned to each candidate variable, we will calculate the adjusted $\log$ coefficient of each candidate variable from the multivariable model, round it to the nearest 0.5 , and then double the rounded $\log$ coefficients to form an integer. ${ }^{26-29}$ We will assess the performance of the clinical prediction rules through internal validation using bootstrapping methodology with repetitions and calculate the area under the receiver operating characteristic (ROC) curve..$^{30-33}$

We will also externally validate the clinical prediction rules derived in Uganda ${ }^{9}$ and in Mozambique ${ }^{10}$ by calculating the sensitivity, specificity and positive and negative likelihood ratios of each score at $\geq$ each specified cut point as defined in these rules. We will also create ROC curves for each existing score. We will assess the association of clinicians' estimated probability of postdischarge outcomes and actual outcomes using ROC curves. We will use multivariable logistic regression to identify factors independently associated with 60-day hospital readmission and unplanned clinic visits among neonates and children. As previous studies suggest that healthcare seeking is infrequent among children who experience postdischarge mortality, ${ }^{34}{ }^{35}$ we will review data from follow-up calls and verbal autopsies to calculate descriptive statistics of the proportion of caregivers who seek care in the postdischarge period and compare rates of care-seeking between children who die in the postdischarge period to those who survived.

\section{ETHICS AND DISSEMINATION}

This study received ethical clearance from the Tanzania National Institute of Medical Research, Muhimbili University of Health and Allied Sciences, the John F. Kennedy Medical Center Institutional Review Board and the Boston Children's Hospital Institutional Review Board. We will disseminate our results in the form of scientific conference presentations and as peer-reviewed publications.

\section{Author affiliations}

${ }^{1}$ Division of Pediatric Emergency Medicine, Emory University School of Medicine, Atlanta, Georgia, USA

${ }^{2}$ Emergency Medicine, Children's Healthcare of Atlanta, Atlanta, Georgia, USA

${ }^{3}$ Department of Pediatrics and Child Health, Muhimbili University of Health and Allied Sciences, Dar es Salaam, United Republic of Tanzania

${ }^{4}$ Department of Pediatrics, John F. Kennedy Medical Center, Monrovia, Liberia

${ }^{5}$ Department of Emergency Medicine, Muhimbili University of Health and Allied

Sciences, Dar es Salaam, United Republic of Tanzania

${ }^{6}$ Department of Nutrition, Harvard T.H. Chan School of Public Health, Boston, Massachusetts, USA

${ }^{7}$ Department of Global Health and Population, Harvard T.H. Chan School of Public Health, Boston, Massachusetts, USA

${ }^{8}$ Pediatric Biostatistics Core, Emory University School of Medicine, Atlanta, Georgia, USA
${ }^{9}$ Division of Emergency Medicine, Boston Children's Hospital, Boston,

Massachusetts, USA

${ }^{10}$ Department of Pediatrics, Harvard Medical School, Boston, Massachusetts, USA

${ }^{11}$ Division of Gastroenterology, Hepatology, and Nutrition, Boston Children's

Hospital, Boston, Massachusetts, USA

${ }^{12}$ Center for Nutrition, Boston Children's Hospital, Boston, Massachusetts, USA

Acknowledgements The authors thank Juliana Mghambha, Mohamed Bakari, Dr. Nodermus Elias, Dr. Lawrence Kurwa, Dr. Tabu Mvile, Dr. Musa Malifedha, Veneranda Ndesangia, Naomi Mmbaga, Christina Japhet, Celestine Kagunila, Agnes Obed, Anna Fundi and Sarah Somji for their assistance in data collection and data management. We thank Melvin M. Kabba-Kay and Fidelis Njokanma for their support of this study. We also thank the nurses and nurse supervisors who have contributed to data collection at both Muhimbili National Hospital and the John F. Kennedy Medical Centre.

Contributors All authors contributed to the study conceptualisation, the study design, sample size calculations and writing of the protocol for this study. CAR, KM and $\mathrm{CD}$ prepared the first draft of the paper. All authors contributed to revisions of the manuscript and contributed to the revision of the final manuscript. All authors have read and approved the final manuscript.

Funding This work was supported by the National Institutes of Health (K24 DK104676and P30 DK040561 to CPD), the Boston Children's Hospital Global Health Program to CAR and the Palfrey Fund for Child Health Advocacy to CAR.

Disclaimer The funders had no role in the study design or in the collection, analysis, or interpretation of the data. The funders did not write the report and had no role in the decision to submit the paper for publication.

Competing interests None declared.

Patient consent for publication Not applicable.

Provenance and peer review Not commissioned; externally peer reviewed.

Data availability statement Data are available upon reasonable request to the corresponding author.

Supplemental material This content has been supplied by the author(s). It has not been vetted by BMJ Publishing Group Limited (BMJ) and may not have been peer-reviewed. Any opinions or recommendations discussed are solely those of the author(s) and are not endorsed by BMJ. BMJ disclaims all liability and responsibility arising from any reliance placed on the content. Where the content includes any translated material, BMJ does not warrant the accuracy and reliability of the translations (including but not limited to local regulations, clinical guidelines, terminology, drug names and drug dosages), and is not responsible for any error and/or omissions arising from translation and adaptation or otherwise.

Open access This is an open access article distributed in accordance with the Creative Commons Attribution Non Commercial (CC BY-NC 4.0) license, which permits others to distribute, remix, adapt, build upon this work non-commercially, and license their derivative works on different terms, provided the original work is properly cited, appropriate credit is given, any changes made indicated, and the use is non-commercial. See: http://creativecommons.org/licenses/by-nc/4.0/.

ORCID iD

Chris A Rees http://orcid.org/0000-0001-6449-0377

\section{REFERENCES}

1 You D, Hug L, Ejdemyr S, et al. Global, regional, and national levels and trends in under-5 mortality between 1990 and 2015 with scenario-based projections to 2030: a systematic analysis by the UN Inter-agency group for child mortality estimation. Lancet 2015;386:2275-86.

2 GBD 2019 Demographics Collaborators. Global age-sexspecific fertility, mortality, healthy life expectancy (HALE), and population estimates in 204 countries and territories, 1950-2019: a comprehensive demographic analysis for the global burden of disease study 2019. Lancet 2020;396:1160-203.

3 Forde I, Tripathi V. Association of place of residence and Under-Five mortality in middle- and low-income countries: a meta-analysis. Children 2018;5:51.

4 Kinney MV, Kerber KJ, Black RE, et al. Sub-Saharan Africa's mothers, newborns, and children: where and why do they die? PLoS Med 2010;7:e1000294.

5 Kagabo DM, Kirk CM, Bakundukize B, et al. Care-seeking patterns among families that experienced under-five child mortality in rural Rwanda. PLoS One 2018;13:e0190739. 
6 Kruk ME, Gage AD, Arsenault C, et al. High-quality health systems in the sustainable development goals era: time for a revolution. Lancet Glob Health 2018;6:e1196-252.

7 Wiens MO, Pawluk S, Kissoon N, et al. Pediatric post-discharge mortality in resource poor countries: a systematic review. PLoS One 2013;8:e66698.

8 Wiens MO, Kissoon N, Kabakyenga J. Smart Hospital discharges to address a neglected epidemic in sepsis in low- and middle-income countries. JAMA Pediatr 2018;172:213-4.

9 Wiens MO, Kumbakumba E, Larson CP, et al. Postdischarge mortality in children with acute infectious diseases: derivation of postdischarge mortality prediction models. BMJ Open 2015;5:e009449.

10 Madrid L, Casellas A, Sacoor C, et al. Postdischarge mortality prediction in sub-Saharan Africa. Pediatrics 2019;143:e20180606.

11 Liu L, Oza S, Hogan D, et al. Global, regional, and national causes of under-5 mortality in 2000-15: an updated systematic analysis with implications for the sustainable development goals. The Lancet 2016;388:3027-35.

12 Villamor E, Misegades L, Fataki MR, et al. Child mortality in relation to HIV infection, nutritional status, and socio-economic background. Int J Epidemiol 2005;34:61-8.

13 Sakita FM, Sawe HR, Mwafongo V, et al. The burden and outcomes of abdominal pain among children presenting to an emergency department of a tertiary hospital in Tanzania: a descriptive cohort study. Emerg Med Int 2018;2018:3982648

14 Moons KGM, Royston P, Vergouwe Y, et al. Prognosis and prognostic research: what, why, and how? BMJ 2009;338:b375.

15 Finocchario-Kessler S, Gautney BJ, Khamadi S, et al. If you text them, they will come: using the HIV infant tracking system to improve early infant diagnosis quality and retention in Kenya. AIDS 2014;28 Suppl 3:S313-21.

16 World Health Organization. Verbal autopsy standards: ascertaining and attributing causes of death, 2016. Available: http://www.who.int/ healthinfo/statistics/verbalautopsystandards/en/ [Accessed 15 Jul 2018].

17 Islam MA, Rahman MM, Mahalanabis D, et al. Death in a diarrhoeal cohort of infants and young children soon after discharge from Hospital: risk factors and causes by verbal autopsy. J Trop Pediatr 1996;42:342-7.

18 Chelo D, Nguefack F, Ntoude A, et al. Verbal autopsy and therapeutic itinerary of children who die before arrival in a paediatric centre in Yaoundé, Cameroon. Transl Pediatr 2016;5:16-22.

19 Chhibber AV, Hill PC, Jafali J, et al. Child mortality after discharge from a health facility following suspected pneumonia, meningitis or septicaemia in rural Gambia: a cohort study. PLoS One 2015;10:e0137095.

20 Ngari MM, Fegan G, Mwangome MK, et al. Mortality after inpatient treatment for severe pneumonia in children: a cohort study. Paediatr Perinat Epidemiol 2017;31:233-42.
21 O'Sullivan NP, Lelijveld N, Rutishauser-Perera A, et al. Follow-Up between 6 and 24 months after discharge from treatment for severe acute malnutrition in children aged 6-59 months: a systematic review. PLoS One 2018;13:e0202053.

22 John $\mathrm{C}$, Diala U, Adah R, et al. Survival and nutritional status of children with severe acute malnutrition, six months post-discharge from outpatient treatment in Jigawa state, Nigeria. PLoS One 2018;13:e0196971.

23 Wiens MO, Kissoon N, Kumbakumba E, et al. Selecting candidate predictor variables for the modelling of post-discharge mortality from sepsis: a protocol development project. Afr Health Sci 2016;16:162-9.

24 Nemetchek BR, Liang LD, Kissoon N, et al. Predictor variables for post-discharge mortality modelling in infants: a protocol development project. Afr Health Sci 2018;18:1214-25.

25 Zou H, Hastie T. Regularization and variable selection via the elastic net. J Royal Statistical Soc B 2005;67:301-20.

26 Reed C, Madhi SA, Klugman KP, et al. Development of the respiratory index of severity in children (RISC) score among young children with respiratory infections in South Africa. PLoS One 2012;7:e27793.

27 Gallagher KE, Knoll MD, Prosperi C, et al. The predictive performance of a pneumonia severity score in human immunodeficiency virus-negative children presenting to hospital in 7 low- and middle-income countries. Clin Infect Dis 2020;70:1050-7.

28 Harrell FE, Lee KL, Mark DB. Multivariable prognostic models: issues in developing models, evaluating assumptions and adequacy, and measuring and reducing errors. Stat Med 1996;15:361-87.

29 Le Gall JR, Lemeshow S, Saulnier F. A new simplified acute physiology score (saps II) based on a European/North American multicenter study. JAMA 1993;270:2957-63.

30 Steyerberg EW, Harrell FE. Prediction models need appropriate internal, internal-external, and external validation. J Clin Epidemiol 2016;69:245-7.

31 Steyerberg EW, Harrell FE, Borsboom GJ, et al. Internal validation of predictive models: efficiency of some procedures for logistic regression analysis. J Clin Epidemiol 2001;54:774-81.

32 Riley RD, Ensor J, Snell KIE, et al. Calculating the sample size required for developing a clinical prediction model. BMJ 2020;368:m441.

33 Iba K, Shinozaki T, Maruo K, et al. Re-evaluation of the comparative effectiveness of bootstrap-based optimism correction methods in the development of multivariable clinical prediction models. BMC Med Res Methodol 2021;21:9.

34 Chisti MJ, Graham SM, Duke T, et al. Post-discharge mortality in children with severe malnutrition and pneumonia in Bangladesh. PLoS One 2014;9:e107663.

35 Lapidot R, Larson Williams A, MacLeod WB, et al. Verbal autopsies for out-of-hospital infant deaths in Zambia. Pediatrics 2021;147:e20201767. 